\title{
Broadband Solid State GaAs Power Amplifier for L and S Bands Applications.
}

\author{
Mohamed Ribate ${ }^{1}$, Rachid Mandry ${ }^{1}$, Larbi El Abdellaoui ${ }^{1}$, Ahmed Errkik ${ }^{1}$, \\ Mohamed Latrach ${ }^{2}$ \\ ${ }^{1}$ LMTEE, FST of Settat, Hassan $1^{\text {st }}$ University, Settat, Morocco. \\ ${ }^{2}$ Microwave Group ESEO Angers, France \\ * Corresponding author's Email: ribate.mohamed@gmail.com
}

\begin{abstract}
This paper proposes the research of a single stage Broadband Solid State Power Amplifier (BPA) based on ATF13786 transistor, using GaAs process. This BPA operates in the frequency band ranging from $1.35 \mathrm{GHz}$ to $3 \mathrm{GHz}$ which covers the mainstream communication standards running in $\mathrm{L}$ and $\mathrm{S}$ Bands. The design approach is based on the real frequency technique. The simulated results are obtained by using ADS circuit simulator. In order to improve the broadband response, a multi-section quarter wave impedance transformer combined with an approximate transformation of a previously designed lumped elements into a transmission lines are used. The simulation results show a saturated output power of $17.85 \mathrm{dBm}$, with PAE of the $11 \%$ and a maximum power gain of $16.68 \mathrm{~dB}$.
\end{abstract}

Keywords: Broadband Power Amplifier, Real Frequency Technique, Solid State, GaAs.

\section{Introduction}

At the present, almost every communication system has some sort of transceiver, and inherently a Radio Frequency Power Amplifier (RFPA), running in one of the following bands (defined by IEEE standard 521-1984): $\mathrm{L}-\mathrm{S}-\mathrm{C}-\mathrm{X}$ or Ku bands, from $1 \mathrm{GHz}$ to $18 \mathrm{GHz}$, operating at Ultra-High Frequencies (UHF) and Super-High Frequencies (SHF) [1-7].

Nowadays, power amplifiers are used everywhere in our life, we are driving cars that are smarter, equipped with new technologies such as parking radar sensor system and GPS. We receive signals from satellites in our home with satellite TV receivers and in our cars or phones from Global Positioning System (GPS) [8-11].

Essentially, Power Amplifiers are critical components for many applications, among which communication systems, Magnetic Resonance Imaging (MRI), semiconductor manufacturing, microwave heating, radars, avionics, medical microwave imaging, electronic warfare and navigation systems represent just a few examples [12-17]. Given such enormously spread fields, power amplifiers sets may greatly differ in technology, design and operating requirements [18]. As a consequence, a widespread architectures of PA have been realized in the past 2 decades, from travelling-wave tube power amplifiers for very high power systems such as radars or satellite payloads, to solid state power amplifiers for medium and low power systems like wireless communication handsets [19].

On the other hand, the improvement in broad bandwidth demands is primarily driven by the next generation of communication systems, which require significantly wide bandwidth. As a result, the interest in the power amplifiers with broad bandwidths and with good performance 
has grown immensely over recent years. Such systems involve stringent requirements from broadband amplifiers in terms of high gain, high stability, high Power Added Efficiency (PAE) and high output power over a wide frequency ranges [20].

The design of broadband power amplifiers is basically equivalent to build an appropriate circuit able to convert DC power supplied to microwave transistor, into Microwave/Radio Frequency power, successively transferred to an external load, in a given band or a range of frequencies of interest [21].

Various approaches, configurations and techniques have been developed and explored for this purpose, trying to meet at the same time several contrasting requirements in terms of gain flatness $v s$ broadband matching, efficiency $v s$ linearity or low distortion $v s$ high output power. Among such configurations of BPA, balanced structures, distributed structures and Doherty configurations that use various matching network schemes as well as reactive filter synthesis, shunt or series feedback and resistive/reactive matching [22].

The reactive/resistive matching technique gives a virtuous power performance and a moderate bandwidth. Thus, the wideband design is difficult. This scheme also has poor VSWR and gain flatness. The shunt/series feedback topology results in lower power density and higher noise figure. However, this configuration is good for VSWR, multi-octave bandwidth and gain flatness. The distributed power amplifiers have the capability of broad bandwidth and achieves excellent gain, but shows weak points in limitation of output power, poor PAE and requiring large area for circuit realization [23].

The work presented in this paper is basically based on the Real Frequency Technique (RFT), in combination with other broadband matching techniques such as multi-section quarter wave impedance transformer, approximate transformation of previously designed lumped element matching circuits into the transmission line matching networks and both single and double stub tuners.

Comparing the matching techniques adopted in this work, with similar contemporary state of the art broadband matching techniques, the RFT is probably the best circuit design available for the design of broadband power amplifiers. The RFT has being pertinent for all matching problems and permits optimizing many performance factors of single or multi-stage RF power amplifiers [24].

In this paper we present the design of a broadband solid state power amplifier, operation in the frequency range from $1.35 \mathrm{GHz}$ to $3 \mathrm{GHz}$ which compromise the mainstream applications and communication standards running in $\mathrm{L}$ and $\mathrm{S}$ bands. The proposed BPA reaches a saturated output power of $17.56 \mathrm{dBm}$ and a maximum power gain of $16.85 \mathrm{~dB}$. The design architecture achieves a simple configuration with an excellent matching performance over $1650 \mathrm{MHz}$ bandwidth.

\section{Broadband Power Amplifier circuit design}

Regardless of its characterization as narrowband, wideband, high power, low noise or else, all power amplifiers have in common feature of converting the DC power supplied to their active devices, into RF power as well as providing a positive power gain at the requested frequency or rang of frequencies.

The design of Broadband Power Amplifier requires an accurate active device, appropriate bias and matching networks, stability during operation and easiness of circuit implementation. The effectiveness of the design approach is evaluated by its aptitude to achieves high output 
power, maximum power gain across a broad bandwidth with minimum stages of PA and under stable operating conditions. The exigency for linearity or high efficiency can be reviewed where it is needed. Keep in mind that, for the stability requirement, it is indispensable to examine the frequency ranges in which the RF transistor may be unstable.

The simplest possible single stage power amplifier consists of a single RF transistor -be it a BJT, FET or otherwise - DC supplied through a DC bias network, and connected through an input and output matching networks, to a source and load respectively, both having same impedance $Z_{0}$, as shown in Figure 1.

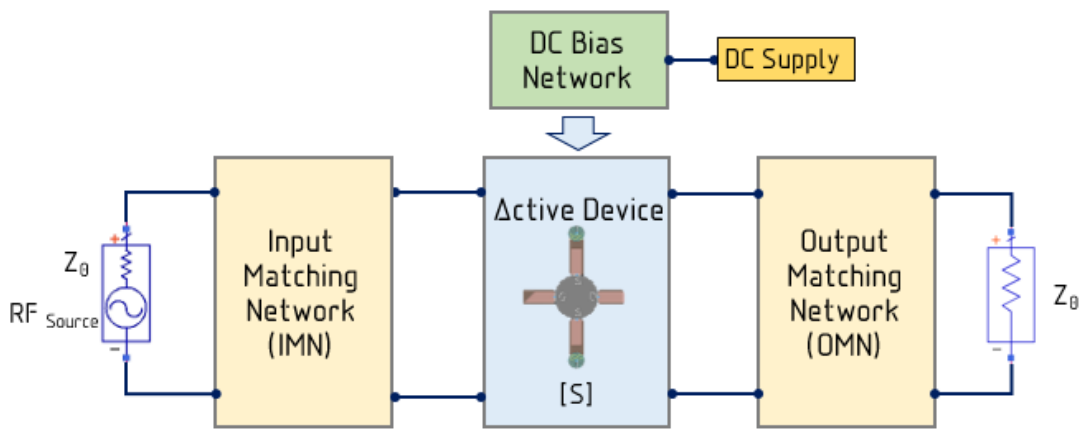

Fig. 1. Single Stage Power Amplifier Block Diagram

At high frequencies, to provide maximum power transfer of the RF power available from the RF source to the load, an accurate input and output matching networks are required as well as a specific DC bias.

\subsection{Biasing circuit of the BPA}

At high frequencies, biasing networks takes an important parts of power amplifiers design, and they must be designed carefully to avoid low frequency oscillations due to high device gain, high capacitance and low substrate resistivity. Despite the biasing parameters depends on the active device technology, the design approaches of biasing circuit are basically the same [25].

In practice, there are many basing schemes available for RF power amplifiers. According to the designer choice, the biasing circuits could be integrated in the matching circuits or they stand-alone.
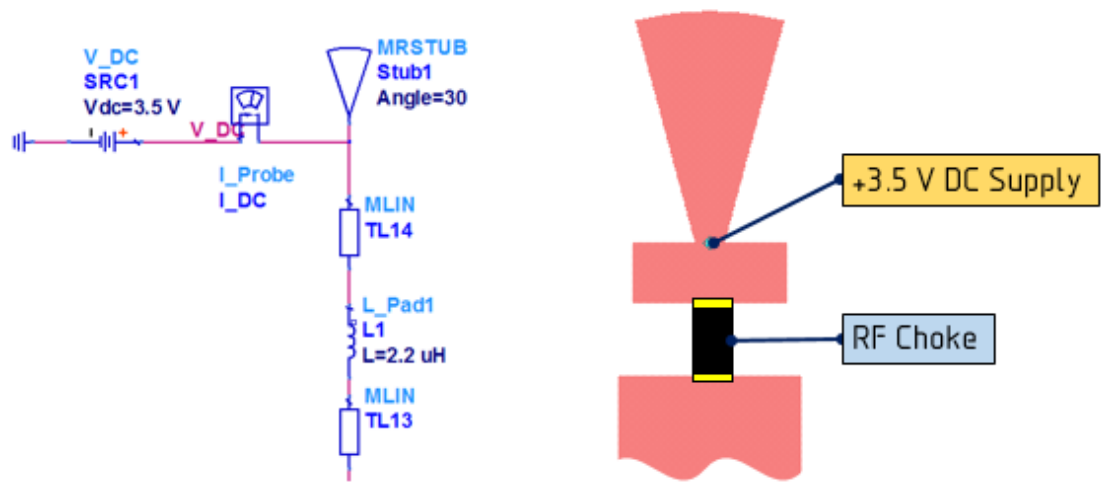

Fig. 2. The proposed microwave biasing circuit. 
However, when the DC bias voltage supplied to the active device, the RF energy must not break through the bias port, otherwise degradation of RF performance in terms of PAE, noise figure and power gain takes place.

Basically, a biasing circuit consists of RF choke and a DC block. The DC block can be either a 3-dB backward -wave coupler or chip capacitors, and must be present a short circuit at the operating frequency. The value of the capacitor must be selected in such a way that $\omega C=$ $2 \pi f C$ is very large.

The configuration of the proposed biasing network is illustrated in Figure 2. The active device -ATF786- is supplied with a single supply voltage VCC of $+3.5 \mathrm{~V}$. the biasing network is synthesized by using a radial line section terminated by a RF choke. This configuration is smaller in size and provides better bandwidth than $\lambda / 4$ open circuited stub.

\subsection{Broadband matching networks design}

The impedance matching networks takes a major part in the design process of the BPA. Basically, the active device is mismatched with the source and the load. Consequently, the RF energy available from the source is not delivered to the load, and this makes a loss of RF energy. In other words, to provide a maximum power transfer of the RF energy from the input port to the active device, from the active device to the output port or between devices, an accurate matching circuit should be designed at the interface between two sections having mismatched impedances, otherwise, a portion of the electrical signal propagated in the circuit will be reflected. In a broad sense, the matching network can be defined as a circuit that transforms a given impedance into a desired impedance.

Essentially, the matching network design depends on the operating frequency of the PA. According to the broadband design approach, there are various impedance matching schemes and techniques that can be deployed to match two given mismatched impedances, regardless of whether the matching is accomplished with transmission lines or lumped elements.

The selection of an appropriate matching technique might not always be clear, however, it is basically determined by the following factors: bandwidth, complexity and implementation.

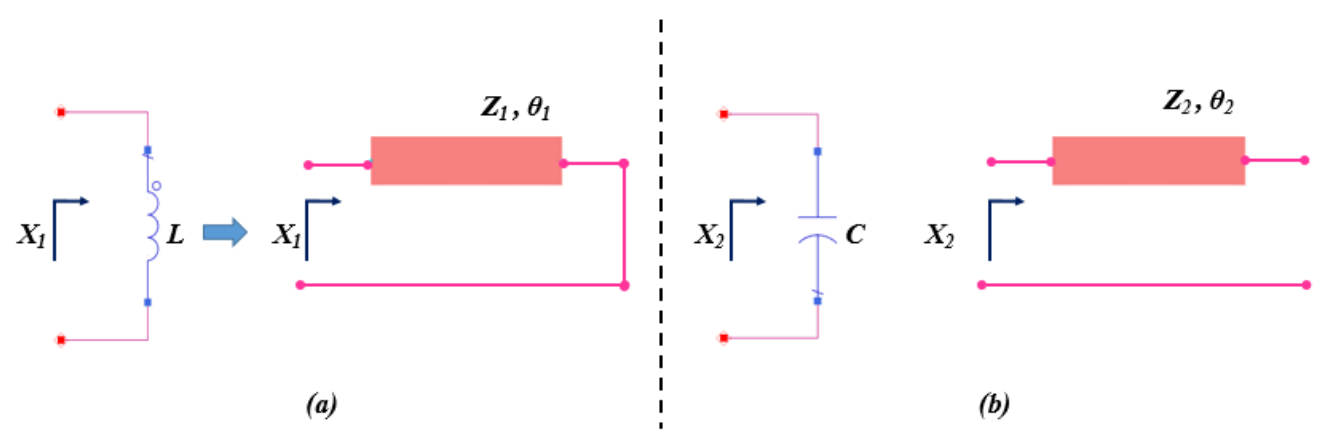

Fig. 3. Equivalence between lumped elements and transmission lines.

In this work, a combination of matching techniques is deployed to achieve the broadband bandwidth of the proposed BPA. The first one is an approximate transformation of lumped elements into transmission lines. At high frequency, excepting an MMIC (Monolithic Microwave Integrated Circuit) design, the implementation of lumped elements such as capacitors and inductors is very difficult where they can be considered as distributed elements. Thus, in this work, the design of the input and output matching networks is based on an 
approximate transformation of lumped elements into transmission lines, which can be established by applying Richard's transformation.

Figure 3 shows the equivalence between lumped elements and transmission lines. The value of $Z_{1}, \theta 1, Z_{2}, \theta 2$ can be obtained by using the following equations:

$$
\begin{aligned}
& X_{1}=Z_{1} \tan \left(\theta_{1}\right) \approx \omega L \\
& X_{2}=\frac{Z_{2}}{2 \tan \left(\theta_{2}\right)}=\frac{1}{\omega C}
\end{aligned}
$$

From the equation (1) and (2), the variables are $Z_{1}, Z_{2}, \theta 1, \theta 2$. As a results, there are two degrees of freedom in implementation of a capacitor or inductor respectively.

The second technique used in the design of the matching networks is the Multi-Section Quarter Wave Impedance Transformer (MSQWIT). Typically, there are two widely known MSQWIT: Chebyshev and Binomial. In this paper we adopt the second transformer. A schematic diagram of a binomial transformer is shown in Figure 4.

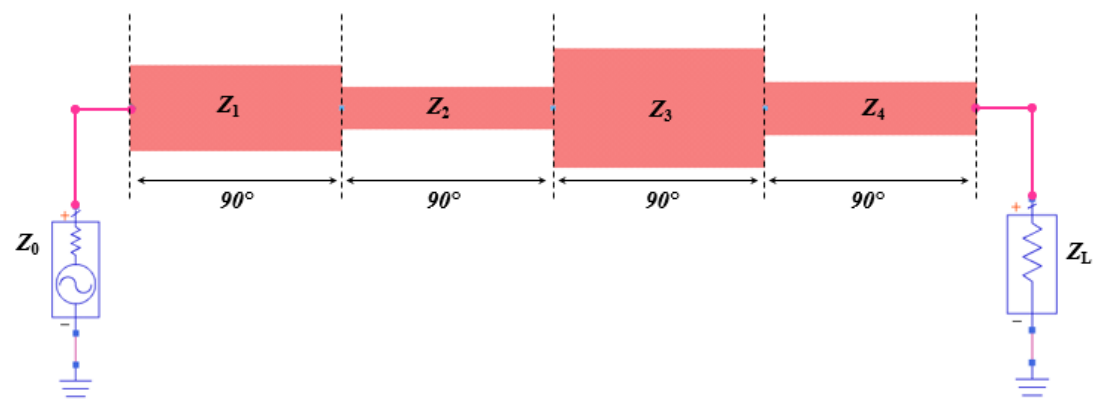

Fig. 4. four-section quarter-wave impedance transformer.

The sections impedances can be calculated approximately by using the following equations:

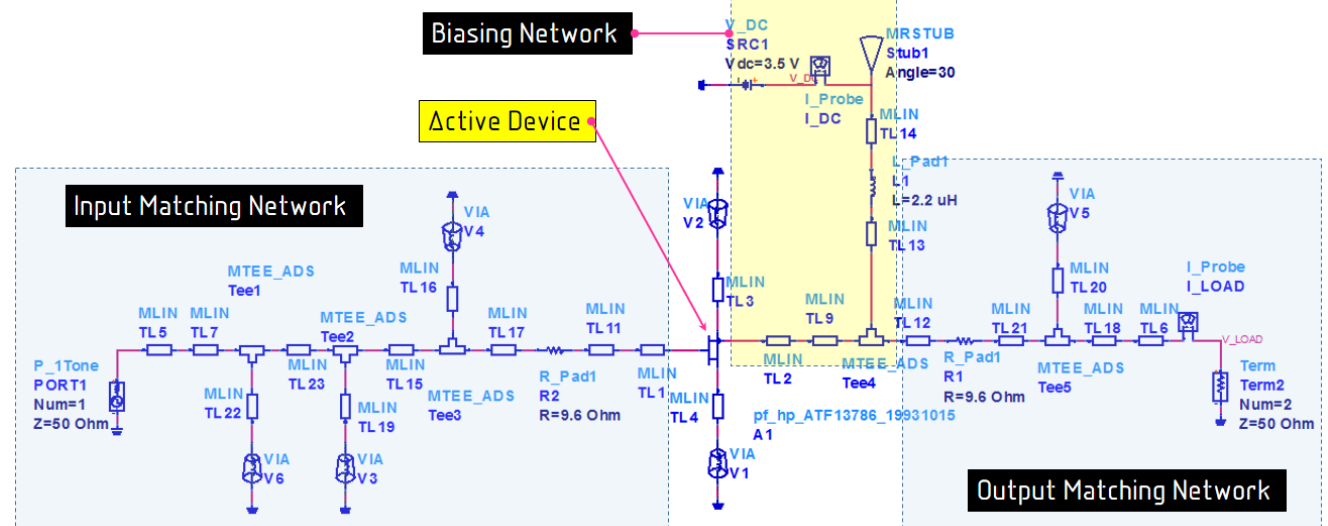

Fig. 5. The proposed single stage BPA.

$$
\ln \frac{Z_{n+1}}{Z_{n}}=2 \rho_{n}=2^{-N} C_{n}^{N} \ln \frac{Z_{L}}{Z_{0}}
$$




$$
C_{n}^{N}=\frac{N !}{(N-n) ! n !}
$$

Where $C_{n}^{N}$ are the binomial coefficients. $Z_{n}$ and $Z_{n+1}$ are the impedances of the $n t h$ and $(n+1) t h$ sections and $\rho n$ is the reflection coefficient of the junction between $Z_{n}$ and $Z_{n+1}$.

By using the concepts mentioned above, the proposed BPA schematic is illustrated in Figure 5 .

\section{Simulation results and discuss}

\subsection{Stability analysis}

According to the stability criterion of PA, the unconditionally stability is achieved when the following Rollett and Bodway equations are simultaneously validated:

$$
\begin{gathered}
k=\frac{1+|\Delta|^{2}-\left|S_{11}\right|^{2}-\left|S_{22}\right|^{2}}{2\left|S_{12} S_{21}\right|}>1 \\
B_{1}=1+\left|S_{11}\right|^{2}+\left|S_{22}\right|^{2}-|\Delta|^{2}>0
\end{gathered}
$$

Where

$$
\Delta=S_{11} S_{22}-S_{12} S_{21}
$$

$\mathrm{K}$ is the Rollett stability factor and $\mathrm{B}_{1}$ is the Bodway stability factor. As shown in Figure 6, the stability requirement of the proposed BPA is established over the operating bandwidth.

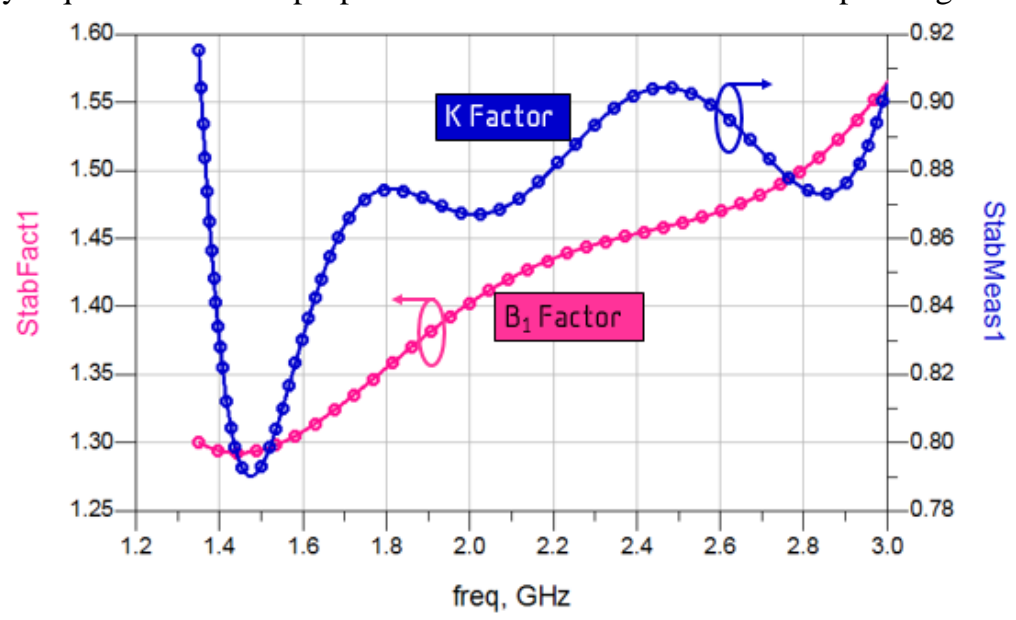

Fig. 6. Curves of $\mathrm{K}$ and $\mathrm{B}_{1}$ Factors versus frequency.

\subsection{Small Signal Simulation}

The RF performance of the proposed BPA is examined using small signal two port scattering parameters simulations which consists of measuring the reflected, transmitted and 
incident waves. We note that the S-parameters simulations are done in $50 \Omega$ source and load impedances. Figure 7 shows the curves of input and output return loss over the bandwidth ranging from $1.35 \mathrm{GHz}$ to $3 \mathrm{GHz}$. As we can see, the maximum value that can be reached by the input return loss $S_{11}$ is $-13 \mathrm{~dB}$, where the output return loss $S_{22}$ reaches a maximum value of $-15 \mathrm{~dB}$ over the operating frequencies. As a result, the good broadband matching of the proposed $\mathrm{BPA}$ is performed.

The internal feedback of the active device is represented by the parameter $S_{12}$. In order to improve the degree of isolation between the output and input as well as the stability of the proposed BPA, it is more required to have $S_{12}$ as small as possible.

The simulated revers isolation $S_{12}$ and small signal gain $S_{21}$ are illustrated in Figure 8. As we can observe, the revers isolation $S_{12}$ reaches a maximum value of $-20 \mathrm{~dB}$. The small signal gain $S_{21}$ varies between a maximum value of $16.78 \mathrm{~dB}$ and a minimum value of $10 \mathrm{~dB}$. Then, for the proposed BPA, we can clearly assume that the unconditionally stability is assured, the broadband impedance matching is accomplished with a good power gain performance over a broad bandwidth of $1650 \mathrm{MHz}$.

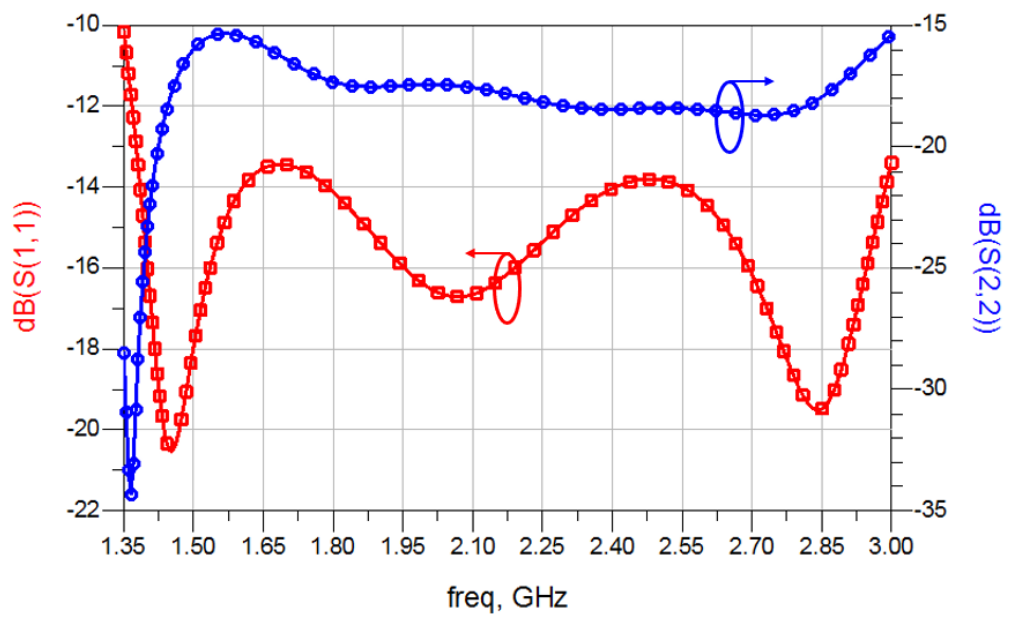

Fig. 7. Curves of $\mathbf{S}_{11}$ and $\mathbf{S}_{\mathbf{2} 2}$ from $1.35 \mathrm{GHz}$ to $3 \mathrm{GHz}$.

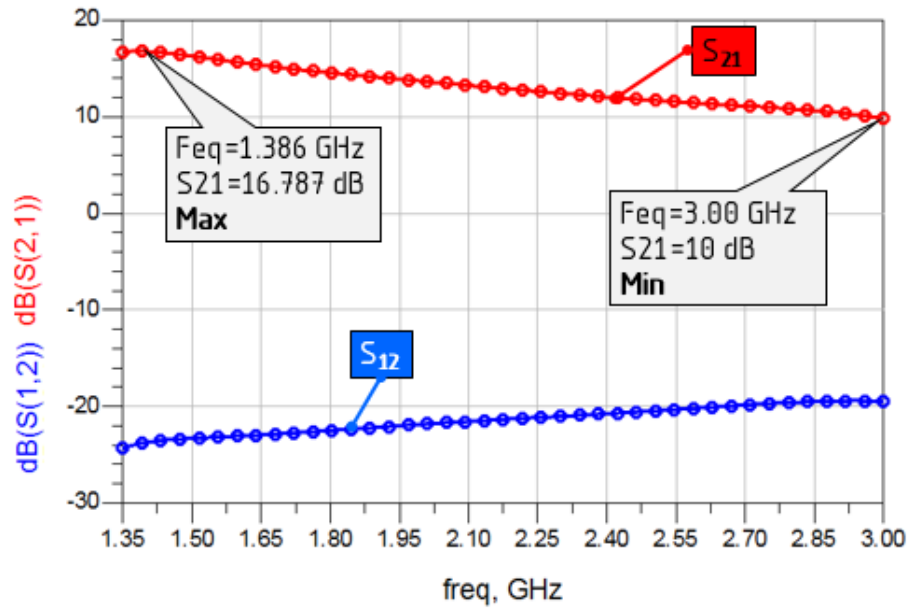

Fig. 8. Curves of $S_{12}$ and $S_{21}$ versus frequency. 


\subsection{Large signal simulation}

From energetic standpoint, a power amplifier can be considered as a device able to convert DC power supplied to the active device, into an RF energy. The efficiency of this conversion is performed by means of Power Added Efficiency (PAE), defined as the ratio between the added power and the supplied DC power, which the added power is the subtraction of the input power from the output power.

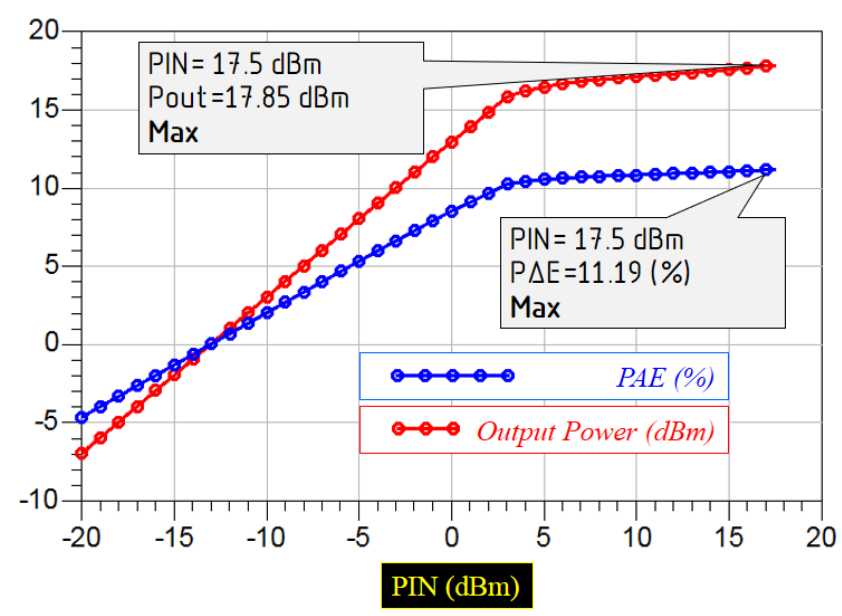

Fig. 9. PAE and Output Power versus frequency.

As shown in Figure 9, at $2.15 \mathrm{GHz}$, the proposed BPA achieves a maximum value of PAE of $11.19 \%$ with a saturated output power of $17.85 \mathrm{dBm}$, thus corresponding to $60.95 \mathrm{~mW}$ of output power.

Table 1. Performance comparison between the proposed PBA and other BPAs

\begin{tabular}{cccccccc}
\hline Ref. & $\begin{array}{c}\text { Freq } \\
{[\mathrm{GHz}]}\end{array}$ & $\begin{array}{c}\text { Psat } \\
{[\mathrm{dBm}]}\end{array}$ & $\begin{array}{c}\text { Gain } \\
{[\mathrm{dB}]}\end{array}$ & $\begin{array}{c}\text { PAE } \\
{[\%]}\end{array}$ & $\begin{array}{c}\text { S11 } \\
{[\mathrm{dB}]}\end{array}$ & $\begin{array}{c}\text { S22 } \\
{[\mathrm{dB}]}\end{array}$ & $\begin{array}{c}\text { Supply } \\
{[\mathrm{V}]}\end{array}$ \\
\hline$[26] 2015$ & $1.9-2.7$ & 28.1 & 11 & 13.7 & - & - & 2.5 \\
{$[27] 2016$} & $1.8-2.8$ & 28 & 25 & 6.1 & $-12 \mid-25$ & $-09 \mid-19$ & 5 \\
{$[7] 2018$} & $1.75-2.15$ & 8 & - & 11.7 & $-11 \mid-22$ & $-13 \mid-19$ & 12 \\
{$[1] 2018$} & $1.1-3$ & 17.14 & 14.9 & 14.9 & $-10 \mid-35$ & $-10 \mid-25$ & 3 \\
This Work & $1.35-3$ & 17.85 & 16.78 & 11.19 & $-10 \mid-24$ & $-15 \mid-34$ & 3.5 \\
\hline
\end{tabular}

By comparing the proposed BPA with the similar contemporary state-of-the-art broadband PAs, we can clearly see that the matching techniques deployed in this work are very sufficient and permits to achieve a broad bandwidth. From Table 1, the proposed BPA exhibits excellent bandwidth, high gain, good output power and excellent matching across the operating bandwidth as well as simple circuitry when it is compared with the other BPAs. 


\section{Conclusion}

This paper introduces a Broadband Power Amplifier operating in the frequency ranges from $1.35 \mathrm{GHz}$ to $3 \mathrm{GHz}$ with simple circuit configuration. The effectiveness of the designed matching networks was verified by the simulation results.

The large signal simulation shows that the proposed BPA reaches a maximum output of $17.85 \mathrm{dBm}$, with a PAE higher than $11 \%$. This BPA is unconditionally stable over the whole operating bandwidth. A power amplifier with those features is more suitable for various microwave applications running in $\mathrm{L}$ and $\mathrm{S}$ bands such as cellular communication standards, medical microwave imaging, digital television broadcasting, avionics and navigation applications represent just a few examples.

\section{References}

[1] Ribate, M. Zbitou, J. Mandry, R. Errkik, A. Latrach, M.: Broadband GaAs FET Power Amplifier for $\mathrm{L}$ and $\mathrm{S}$ Bands Applications. International Journal of Intelligent Engineering and Systems. Vol. 11 (5), pp. 96-105 (2018).

[2] Ribate, M. Mandry, R. Latrach, M. Errkik, A. El Abdelaoui, L.: GaAs FET Broadband Power Amplifier for L and S Bands Applications, In Proceeding of The $2^{\text {nd }}$ International Conference on Computing and Wireless Communication Systems (ICCWCS, Morocco (2017).

[3] Watkins, G. Wang, S.: The impact of power amplifier turn-on characteristics in cognitive radio networks. Microwave Journal. Vol. 57 (2), pp. 86-92 (2014).

[4] Fang, J. Moreno, J. Quaglia, R. Camarchia, V. Pirola, M. Guerrieri, SD. Ramella, C. Ghione, G.: $3.5 \mathrm{GHz}$ WiMAX GaN Doherty power amplifier with second harmonic tuning. Microwave and Optical Technology Letters. Vol. 54 (11), pp. 2601-2605 (2012).

[5] IEEE Std 521-1984, IEEE Standard Letter Designations for Radar-Frequency Bands, (1984)

[6] Wagner, R.: $3 \mathrm{G}$ power amplifiers: moving from GaAs to CMOS. Microwave Journal. Vol. 55(4), pp. 44-48 (2012).

[7] Rachakh, A. El Abdellaoui, L. Zbitou, J. Errkik, A. Tajmouati, A. Latrach, A.: A Novel Configuration of a Microstrip Microwave Wideband Power Amplifier for Wireless Application. Telkomnika. Vol. 16 (1), pp. 2014-2031 (2018).

[8] Khannur, PB. Chen, X. Yan, DL. Shen, D. Zhao, B. Raja, MK. Sindunata, R. Yeoh, GW. Singh, R.: Universal UHF RFID reader IC in $0.18-\mu \mathrm{m}$ CMOS technology. IEEE Journal Solid-State Circuits. Vol. 43(5), pp. 1146-1155 (2008).

[9] Osepchuck, JM.: Microwave Power Applications. IEEE Transactions on Microwave Theory and Techniques. Vol. 50 (3), pp. 975-985 (2002).

[10] Sobol, H. Tomiyasu, K. Milestones of microwaves.: IEEE Transactions on Microwave Theory and Techniques. Vol. 50 (3), pp. 594-611 (2002).

[11] Kopp, B.A. Borkowski, M. Jerinic, G.: Transmit/receive modules. IEEE Transactions on Microwave Theory and Techniques. Vol. 50 (3), pp. 827-834 (2002).

[12] Willert-Porada, M.: Advances in Microwave and Radio Frequency Processing. Report from the 8th International Conference on Microwave and High Frequency Heating. Springer-Verlag, Germany (2001). 
[13] Osepchuk, J.M.: A history of microwave heating applications. IEEE Transactions on Microwave Theory and Techniques. Vol. 32 (9), pp. 1200-1224 (1984).

[14] Rosen, A. Stuchly, M. A. Vorst, A. V.: Applications of RF/Microwaves in Medicine. IEEE Transactions on Microwave Theory and Techniques. Vol. 50 (3), pp. 963-974 (2002).

[15] Rekanos, I. T. Räisänen, A.: Microwave Imaging in the Time Domain of Buried Multiple Scatterers by Using an FDTD-Based Optimization Technique. IEEE Transactions on Magnetics. Vol. 39 (3), pp. 1381-1384 (2003).

[16] Kaynak, M. Tekin, I. Gurbuz, Y.: Fully integrated low-power SiGe power amplifier for biomedical applications. IET Microwaves Antennas \& Propagation. Vol. 5 (2), pp. 214 219 (2011).

[17]Fear, E.C. Li, X. Hagness, S.C. Stuchly, M.A.: Confocal microwave imaging for breast cancer detection: localization of tumors in three dimensions. IEEE Transaction on Biomedical Engineering. Vol. 49 (8), pp. 812-822 (2002).

[18] Poole, C. Darwazeh, I.: Microwave Active Circuit Analysis and Design. Elsevier Ltd, UK (2016).

[19] Colantonio, P. Giannini, F. Limiti, E.: High Efficiency RF and Microwave Solid State Power Amplifiers. John Wiley \& Sons, Ltd, UK (2009).

[20] Virdee, B.S. Virdee, A.S. Banyamin, B.Y.: Broadband Microwave Amplifiers. Artech House, Inc, US (2004).

[21] Eroglu, A: Introduction to RF Power Amplifier Design and Simulation. CRC Press, US (2016).

[22] Wu, H. Wang, L. Zhou, P. Ma, J.: A 0.1-1.2 GHz CMOS Ultra-Broadband Power Amplifier. In Proceeding of 2014 IEEE MTT-S International Microwave Symposium, USA (2014).

[23] Inder Babel, Prakash Bhartia.: Microwave Solid State Circuit Design. 2 $2^{\text {nd }}$ Edition, John Wiley \& Sons, Inc, (2003)

[24] Jarry, P. Beneat, J.N.: Microwave Amplifier and Active Device Design Using the Real Frequency Technique. John Wiley \& Sons, Inc, Hoboken, New Jersey (2016).

[25] Raghavan, A. Srirattana, N. Laskar, J.: Modeling and Design Techniques for RF Power Amplifiers. John Wiley \& Sons, Hoboken, New Jersey (2008).

[26] Francois, B. Reynaert, P.: Highly linear fully integrated wideband RF PA for LTEadvanced in 180-nm SOI. IEEE Transactions on Microwave Theory and Techniques. Vol. 63 (3), pp. 649-658 (2015).

[27] Chen, C.Q. Hao, M.L. Li, Z.Q. Du, Z.B. Yang, H.: A 1.8-2.8 GHz Highly Linear Broadband Power Amplifier for LTE-A Application. Progress in Electromagnetics Research C. Vol. 66, pp. 47-54 (2016). 